\title{
Améliorer la compétitivité du bois de sciage légal en provenance de la zone agroforestière au Cameroun
}

\author{
Romain Kana, Norbert Sonne, Barthelemy Ondua (1), \\ Patrick Tadjo (2), Benjamin Ondo (3)
}

(I) WWF, Cameroun

(2) Ministère des forêts et de la faune, Cameroun

(3) ONG de développement local CEPFILD, Cameroun

\begin{abstract}
Since 1997, the year the first community forest in Cameroon was created, sawn wood from community forests has been facing difficulties to find a place in an expanding domestic market. The low competitiveness of community forest products is among the major obstacles to have them help reduce rural poverty as desired in the new forest policies in Central Africa. This article, from the work of the World Wide Fund for Nature team and organizations partners, identifies the factors explaining the low competitiveness of community forests in the domestic market and proposes by way of conclusion some strategies whose implementation will allow community forestry to play its full role in the supply of domestic markets, improving sustainable management of the agroforestry landscape and fostering poverty reduction in rural areas.
\end{abstract}

Key words: wood value chain, artisanal logging, forest policy, community forests

Depuis I997, année d'attribution de la première forêt communautaire au Cameroun, le bois de sciage artisanal, issu des forêts communautaires, a du mal à trouver de la place dans le marché domestique pourtant en pleine expansion. La faible compétitivité de l'offre des forêts communautaires est un des freins importants pour la réduction de la pauvreté en milieu rural telle que souhaitée dans les nouvelles politiques forestières en Afrique Centrale. Le présent article, issu des travaux d'une équipe du Fonds Mondial pour la Nature et des structures partenaires, met en évidence les facteurs explicatifs de la faible compétitivité des forêts communautaires sur le marché domestique et propose en guise de conclusions, quelques stratégies dont la mise en œuvre permettra à la foresterie communautaire de jouer pleinement son rôle dans l'approvisionnement des marchés domestiques, la gestion durable des ressources agroforestières et la réduction de la pauvreté en milieu rural.

Mots clés: filière bois, exploitation artisanale des forêts, politiques forestières, forêts communautaires 


\section{Introduction}

Depuis le sommet de Rio (I992) et la Déclaration de Nations Unies sur les droits des peuples indigènes (2007), le rôle essentiel des peuples autochtones et des communautés locales dans la gestion environnementale et le développement des ressources forestières est mondialement reconnu. En conséquence, les législations forestières de la plupart des pays d'Afrique centrale prévoient l'affectation d'un espace forestier aux collectivités locales décentralisées et aux communautés riveraines. Pays pionnier dans la région, le Cameroun a intégré dans sa nouvelle loi forestière de I994 plusieurs instruments de gouvernance décentralisée dont la foresterie communautaire, en vue entre autres de contribuer à la réduction de la pauvreté en milieu rural (Cuny, 20II).

La Loi forestière camerounaise de I994 offre aux communautés rurales l'opportunité d'acquérir un maximum de 5.000 ha d'espace dans le domaine forestier non permanent (DFnP), pour une exploitation durable du bois et des produits forestiers non ligneux (PFNL). Depuis 1997, année d'attribution de la première forêt communautaire, plus de 500 initiatives de forêt communautaire (FC) bénéficiaires d'au moins une convention avec l'Etat ont été répertoriées au Cameroun. Elles représentent environ I, 5 millions d'hectares dans le domaine agroforestier (MINFOF, 2012). Celles-ci sont appuyées généralement par des structures partenaires du gouvernement, telles que le Fonds Mondial pour la nature (WWF) et le Cercle de promotion des forêts et des initiatives locales de développement (CEPFILD) qui promeuvent actuellement la démarche d'entreprise communautaire auprès d'une cinquantaine d'initiatives de FC (Sonne et al., 20II ; Kana et al., 20II; Bakouma \& Sève, 20I2).

Depuis 2000, la pauvreté a très peu reculé en milieu rural où plus de la moitié de la population continue de vivre en dessous du seuil de pauvreté (ECAM I, I998; ECAM 2, 2003; ECAM 3, 2008), compromettant ainsi l'atteinte des Objectifs du millénaires pour le développement à l'horizon 2015 par le gouvernement camerounais. Jusqu'à ce jour, la contribution des FC à l'éradication de la pauvreté en milieu rural a été très peu significative. A peine $40 \%$ des FC sont vraiment opérationnelles (Ingram et al., 2010), tandis que lorsqu'elles le sont, le taux d'exploitation du bois qui est le principal produit commercialisé se situe en dessous des 30\% du volume annuel qui leur est autorisé (Adja \& Tayou, 2007 ; Nzoyem et al., 20I0). Il existe plusieurs tentatives d'explication de cette faible performance des FC. Selon Lescuyer (2005), le modèle organisationnel des FC, exigé par les pouvoirs publics, ne s'intègre pas aux systèmes locaux de gestion de ressources forestières, suscitant ainsi des conflits qui paralysent le fonctionnement des entités de gestion. Dans le même sciage, Julve et al. (2007) ont mis en évidence la complexité technique des normes de gestion et la forte bureaucratie du processus d'autorisation d'exploitation. Plus récemment, Nzoyem et al. (2010) ont soulevé la faible compétitivité des FC comparativement au secteur informel qui est la principale source d'approvisionnement d'une demande nationale de bois de sciage artisanal en très forte croissance (Cerruti et Lescuyer, 20II). Notre étude s'est penchée sur ce dernier aspect du problème de performance de FC sous un angle marketing, avec pour objectif principal de déterminer les facteurs explicatifs de la faible compétitivité des FC sur le marché national. Pour atteindre cet objectif principal, le présent article devrait répondre à trois questions : 
- Quelles sont les caractéristiques du marché intérieur de bois de sciage artisanal?

- Quels sont les principaux déterminants de compétitivité d'un producteur de bois de sciage artisanal dans le marché intérieur?

- Quelle est la position de l'offre des FC par rapport à ces déterminants de compétitivité?

\section{Méthodologie}

Quelques notions clés de l'analyse de compétitivité

Produit et gamme : généralement, le produit est perçu comme un assemblage d'éléments tangibles sous une certaine forme. Dans le cadre de cette étude nous allons nous inspirer des courants de pensée fondamentale du marketing, en définissant le produit comme un ensemble de caractéristiques tangibles et symboliques dans lequel un acheteur ou consommateur peut trouver une source de satisfaction de ses besoins ou désirs (Stanton, 1964; Lendrevie \& Lindon, 2000). La gamme représente un ensemble de produits satisfaisant les mêmes besoins à la base, mais présentant de légères différenciations. Si ces différenciations sont des déterminants de la qualité du produit, alors le croisement entre ces déterminants de la qualité et les prix permettront de différencier sur le marché les stratégies de haut de gamme, de moyen de gamme et de bas de gamme.

Marché : le marché a plusieurs sens suivant qu'on se situe dans les sciences économiques ou de gestion. Dans la même logique que Lendrevie et Lindon (2000), le concept de marché sera utilisé ici pour caractériser l'importance, la structure et l'évolution des ventes du produit faisant l'objet de notre étude : le bois de sciage artisanal.

Compétitivité : la compétitivité est un terme essentiel dans l'approche d'un marché. Elle explique très souvent pourquoi, une entreprise n'arrive pas à s'épanouir dans un marché pourtant très florissant. Selon Lambin et Chumpitaz (2002), l'analyse de la compétitivité aura pour objectifs d'identifier les concurrents et de déterminer leur supériorité relative sur le marché. Cette supériorité relative peut reposer sur deux aspects, concourant à un avantage concurrentiel : la qualité distinctive du produit et le coût de production. La qualité permet de pratiquer des prix supérieurs à ceux des concurrents, tandis que la production à un coût bas permet à l'entreprise d'être rentable et de résister aux baisses de prix sur le marché. Une entreprise ne peut résister durablement à la concurrence dans un marché que lorsqu'elle a soit un avantage coût, soit un avantage qualité.

Chaîne de valeur : la chaîne de valeur est une notion très utilisée en stratégie d'entreprises et plus particulièrement en stratégie de concurrence. Elle désigne un ensemble d'activités permettant de délivrer un produit ou une valeur aux acheteurs (Kotler et al., 2006). L'analyse de la chaîne de valeur d'un produit a pour finalité d'identifier tous les participants, les activités et leur contribution à la valeur du produit, permettant ainsi de déterminer les sources d'avantages concurrentiels en termes de qualité et/ou en termes de coût. 
La collecte de données

Les données de cette étude ont été obtenues à partir d'un échantillon de douze FC, des opérateurs du secteur du bois de sciage artisanal (exploitants de dépôts de bois et exploitants d'unités de deuxième transformation de sciage artisanal pour l'exportation), et sur la base de l'exploitation de données documentaires existantes dont les rapports d'études préalablement réalisées dans le secteur bois par des organismes tels que: le Centre International pour la Recherche en Foresterie (CIFOR), le Centre pour l'environnement et le Développement (CED) et la Société Néerlandaise de Développement (SNV).

Douze FC, encadrées techniquement par le WWF et des ONGs locales partenaires (CEPFILD et l'OPFCR), ont été les principales cibles de cette étude. Elles sont localisées, tel que l'indique le tableau I ci-dessous, dans trois Départements de la Région du Sud Cameroun: Le Dja et Lobo, l'Océan et la Vallée du Ntem. L'exploitation de leurs documents de gestion ainsi que des fiches de chantiers nous ont permis d'avoir des données sur les coûts de production. Par ailleurs, l'utilisation des données d'inventaires ligneux, contenus dans leur Plan Simple de Gestion (PSG), ont permis d'apprécier le volume et la qualité d'essences de bois disponibles dans les forêts. Pour des raisons d'indisponibilité du gestionnaire, nous n'avons malheureusement pas pu entrer en possession du PSG de la FC ANGONOBO à temps pour l'intégrer dans nos analyses.

\begin{tabular}{|c|c|c|}
\hline Forêts communautaires & Arrondissement & Département \\
\hline \multirow{2}{*}{ ABAPY; APMI ; Solidarité } & Mintom & \multirow{5}{*}{ Dja et Lobo } \\
\hline & Oveng & \\
\hline \multirow[t]{2}{*}{ ANGONOBO-TYE } & Ngoulemakong & \\
\hline & Meyomessala & \\
\hline GICAN II & Sangmélima & \\
\hline APM ; ACOMIME ; ALMA & Ma'an & Vallée du Ntem \\
\hline UCFA; RDFCAB ; OTONON & Akom II & Océan \\
\hline
\end{tabular}

Tableau 1: Les forêts communautaires cibles et leur localisation

La rencontre avec les opérateurs a eu lieu dans 5 villes de commercialisation de bois : Ebolowa, Sangmélima, Kribi, Douala et Yaoundé. Les trois premières sont des villes de proximité des I2 FC présentées plus haut. Par ailleurs, elles sont les principaux points de transit et de consommation du bois produit dans la Région du Sud. Les villes de Douala et Yaoundé sont les deux grands centres urbains du Cameroun. Selon les études antérieures (Plouvier et al., 2002 ; Cerruti \& Lescuyer, 20II), ces deux centres urbains consomment plus de la moitié du sciage artisanal produit au Cameroun, et servent de point de transit pour l'approvisionnement d'autres villes.

Les interviews auprès de $4 \mathrm{I}$ opérateurs tels que répartis dans le tableau 2 ci-dessous, avaient pour objectif de recueillir des informations permettant de comprendre et de décrire le fonctionnement du marché. 


\begin{tabular}{|c|c|c|c|c|c|c|}
\hline Type d'opérateurs & 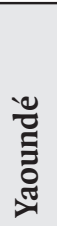 & $\frac{\pi}{\tilde{\Xi}}$ & 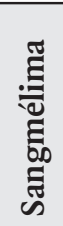 & $\begin{array}{l}\frac{\pi}{3} \\
\frac{0}{0} \\
\text { 퐁 }\end{array}$ & 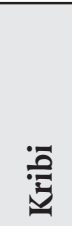 & 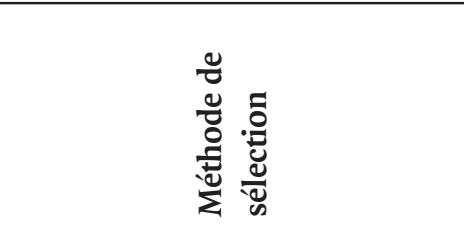 \\
\hline $\begin{array}{l}\text { Exploitants de dé- } \\
\text { pôts de bois sciage } \\
\text { artisanal et déchets } \\
\text { de scieries }\end{array}$ & 12 & 10 & 1 & 3 & 4 & $\begin{array}{l}\text { Recensement des exploitants de } \\
\text { dépôts de bois dans les villes de } \\
\text { Kribi, Sangmélima et Ebolowa } \\
\text { Choix selon la disponibilité et } \\
\text { le profil recherché des vendeurs } \\
\text { dans les principaux dépôts de } \\
\text { bois des villes de Douala et de } \\
\text { Yaoundé }\end{array}$ \\
\hline $\begin{array}{l}\text { Exploitants d'unités } \\
\text { de deuxième } \\
\text { transformation de } \\
\text { sciage artisanal pour } \\
\text { l'export }\end{array}$ & & & 1 & 3 & 7 & Recensement des unités \\
\hline
\end{tabular}

Tableau 2 : Profil et répartition des opérateurs bois interviewés par localités

Dans les villes de Sangmelima, Ebolowa et Kribi, nous avons effectué un recensement des points de vente et unités de deuxième transformation, et procédé à l'interview sur place des opérateurs rencontrés. S'agissant des villes de Yaoundé ou Douala, nous avons effectué des descentes dans certains des principaux dépôts et points de vente de bois connus selon des études antérieures (Plouvier et al., 2002 ; Cerruti \& Lescuyer, 20II) à savoir Tractafric, Camp Yabassi, Mkomo, Ngousso et Nsam.

Dans la mesure où nous collections les données sur des activités de type commercial (prix de ventes, coûts d'approvisionnement...) où il y a une certaine méfiance et une tendance à la rétention d'information en raison de leur importance stratégique selon l'opérateur, nous avons usé de deux approches. La première était d'avoir dans chaque ville un point d'entrée qui était une personne ressource pour le secteur (responsables d'association de vendeurs de bois de sciage, agents du MINFOF, opérateurs réputés ...) et qui se chargeait de nous introduire auprès des opérateurs dans les points de vente afin d'instaurer un minimum de confiance et de volonté à coopérer. Avec cela nous avons essayé, autant que possible, de diversifier le profil des personnes rencontrées (durée dans le secteur, zone d'approvisionnement) afin de diversifier et recouper suffisamment l'information pour s'assurer de leur viabilité. La deuxième approche était de faire de des observations dans les points de vente et d'assister de façon passive aux négociations entre vendeurs et acheteurs sur les prix de vente.

Les données documentaires quant à elles nous ont permis d'une part, d'avoir les informations sur les ventes nationales du bois de sciage artisanal, les coûts de produc- 
tion chez les opérateurs de sciages informels; et d'autre part de confronter nos données sur les circuits de distribution. Elles ont également contribué à expliquer certains faits constatés à l'issue de nos analyses de données.

\section{Résultats et discussions}

\section{Aperçu du marché du bois de sciage artisanal au Cameroun}

Dans ce premier paragraphe des résultats de notre étude, nous allons présenter brièvement le marché du bois de sciage artisanal à travers 4 principales caractéristiques : les produits, le niveau des ventes, les circuits de commercialisation et les principaux acteurs.

En fonction des dimensions des débités et de leur usage, on distingue généralement 6 principaux produits achetés dans le marché intérieur du bois camerounais de sciage artisanal. Ces produits sont présentés dans le tableau 3 ci-dessous.

\begin{tabular}{|c|c|c|c|}
\hline Nom du produit & Dimensions & Usages & Principaux acheteurs \\
\hline $\begin{array}{l}\text { Planche de } \\
\text { menuiserie }\end{array}$ & $\begin{array}{l}\text { Longueur: } 2,20 \mathrm{~m} \\
\text { Largeur: } 0,40 \mathrm{~m} \\
\text { ou } 0,20 \mathrm{~m} \\
\text { Epaisseur: } 0,04 \mathrm{~m} \\
\text { (ou 0,05 m) }\end{array}$ & $\begin{array}{l}\text { Fabrication de meubles, } \\
\text { portes, cadres de fenê- } \\
\text { tres et portes, plafonds, } \\
\text { planchers et carrosseries } \\
\text { de camion. }\end{array}$ & $\begin{array}{l}\text { Menuiserie, } \\
\text { artisans ou } \\
\text { techniciens du } \\
\text { bâtiment }\end{array}$ \\
\hline $\begin{array}{l}\text { Planche de } \\
\text { coffrage }\end{array}$ & $\begin{array}{l}\text { Longueur: } 5 \mathrm{~m} \\
\text { Largeur: 0,30 } \mathrm{m} \\
\text { (ou 0,20 m) } \\
\text { Epaisseur: } 0,03 \mathrm{~m} \\
\text { (ou } 0,02 \mathrm{~m} \text { ) }\end{array}$ & $\begin{array}{l}\text { Travaux de construction } \\
\text { dont: pose de } \\
\text { charpentes, dalles, et } \\
\text { chaînage }\end{array}$ & $\begin{array}{l}\text { Artisans et techniciens } \\
\text { du bâtiment }\end{array}$ \\
\hline Bastaing & $\begin{array}{l}\text { Longueur : } 5 \mathrm{~m} \\
\text { Largeur : 0,17 m } \\
\text { (ou 0,15 m) } \\
\text { Epaisseur : 0,09 m } \\
\text { (ou 0,03 m) }\end{array}$ & $\begin{array}{l}\text { Travaux de constructions } \\
\text { notamment les } \\
\text { charpentes, les poteaux } \\
\text { d'angles. }\end{array}$ & $\begin{array}{l}\text { Artisans et techniciens } \\
\text { du bâtiment }\end{array}$ \\
\hline Chevron & $\begin{array}{l}\text { Longueur: } 5 \mathrm{~m} \\
\text { Largeur: } 0,08 \mathrm{~m} \\
\text { Epaisseur: } 0,08 \mathrm{~m}\end{array}$ & $\begin{array}{l}\text { Travaux de constructions } \\
\text { notamment les } \\
\text { charpentes. }\end{array}$ & $\begin{array}{l}\text { Techniciens et } \\
\text { artisans du bâtiment, } \\
\text { Ménages }\end{array}$ \\
\hline Lattes (I) & $\begin{array}{l}\text { Longueur: } 5 \mathrm{~m} \\
\text { Largeur: } 0,08 \mathrm{~m} \\
\text { Epaisseur: } 0,04 \mathrm{~m}\end{array}$ & $\begin{array}{l}\text { Travaux de constructions } \\
\text { notamment les } \\
\text { charpentes }\end{array}$ & $\begin{array}{l}\text { Techniciens et } \\
\text { artisans du bâtiment, } \\
\text { Ménages }\end{array}$ \\
\hline Madriers (2) & $\begin{array}{l}\text { Plusieurs dimensions } \\
\text { dont: } \\
\text { Longueur : } 3 \mathrm{~m} \\
\text { Largeur : 0,205 } \mathrm{m} \\
\text { Epaisseur : 0,05 m }\end{array}$ & $\begin{array}{l}\text { Travaux de } \\
\text { constructions, grands } \\
\text { chantiers }\end{array}$ & $\begin{array}{l}\text { Techniciens de } \\
\text { bâtiments et grands } \\
\text { travaux }\end{array}$ \\
\hline
\end{tabular}

Tableau 3 : Les principaux produits du sciage artisanal sur le marché (Source : données de notre étude)

(1) Par souci de réduction des coûts de production et de transport, ceux-ci arrivent souvent sur le marché sous forme de cheurons, lesquels vont être redimensionnés en lattes).

(2) Produit rare dans les points de ventes en raison de sa destination principale qui est le circuit de l'exportation. Les dimensions varient en fonction des commandes. 
Estimées à $305.000 \mathrm{~m}^{3}$ de débités en 2002 (Plouvier et al., 2002), les ventes de sciage artisanal auraient plus que triplé en moins de dix ans. Elles sont estimées aujourd'hui à près de I.I00.000 $\mathrm{m}^{3}$ de débité sur les marchés de Yaoundé, Douala et Bertoua (Lescuyer et al., 2009), avoisinant ainsi les ventes du secteur industriel. Les ventes sont tributaires du dynamisme de la demande urbaine, en particulier les grandes villes que sont Yaoundé et Douala. La demande est satisfaite en grande partie par l'exploitation illégale. Elle approvisionnerait à $73 \%$ les marchés de Yaoundé, Douala et Bertoua et à Ioo\% le marché du Grand Nord. Les trois villes, précédemment citées, compteraient aujourd'hui plus de I400 vendeurs de bois (Lescuyer et al., 2009). Le relevé des prix que nous avons effectué sur les marchés montre que la planche artisanale qui était vendu à FCFA 2.000 (environ $€_{3}$ ) à Yaoundé en 2002, s'achète en 20II à FCFA 4.000 (environ €6), soit une augmentation de $100 \%$ des prix en moins de ro ans.

Comme indiqué dans la figure I ci-dessous, le flux des sciages artisanaux du lieu de production jusqu'à l'utilisateur peut nécessiter, en fonction de l'acheteur et du marché visé, 3 types de circuits d'écoulement, lesquels font intervenir plusieurs types d'acteurs dans trois niveaux de la chaîne de mise en marché du bois.

Le circuit 1 est un circuit de commercialisation ultra court, très souvent utilisé dans les petites villes et zones rurales pour satisfaire les demandes des petites menuiseries, des ménages et des petits exportateurs installés dans la ville. Toutes les 2 forêts communautaires enquêtéés utilisent ce circuit. Nous avons recensé II unités de transformation et exportation du bois des forêts communautaires dans les villes de Kribi, Sangmélima et Ebolowa. Le circuit 2 est un circuit court, fonctionnel dans les grandes zones de consommation de bois pour approvisionner une demande importante plus ou moins régulière. Nous avons recensé 8 exploitants de dépôts de bois qui approvisionnent les consommateurs des villes de Kribi, Sangmélima et Ebolowa. Le circuit 3 est un circuit long où il existe un marché de bois à dimension nationale et régionale approvisionnant les marchés de bois d'autres villes. Les grands marchés de bois de Yaoundé fonctionnent dans cette logique en ravitaillant par exemple les marchés de bois de Douala et ceux de la partie septentrionale du Cameroun (Koffi, 2005). Les études du CIFOR (Centre International pour la Recherche en Foresterie) dans la partie méridionale du Cameroun estiment à près de 44.000 le nombre de personnes ayant une activité au niveau production et commercialisation du bois de sciage artisanal (Cerruti \& Lescuyer, 20II). Par ailleurs, celles-ci ont recensé en 20IO, I.219 dépôts de bois dans les seules villes de Yaoundé et de Douala. 


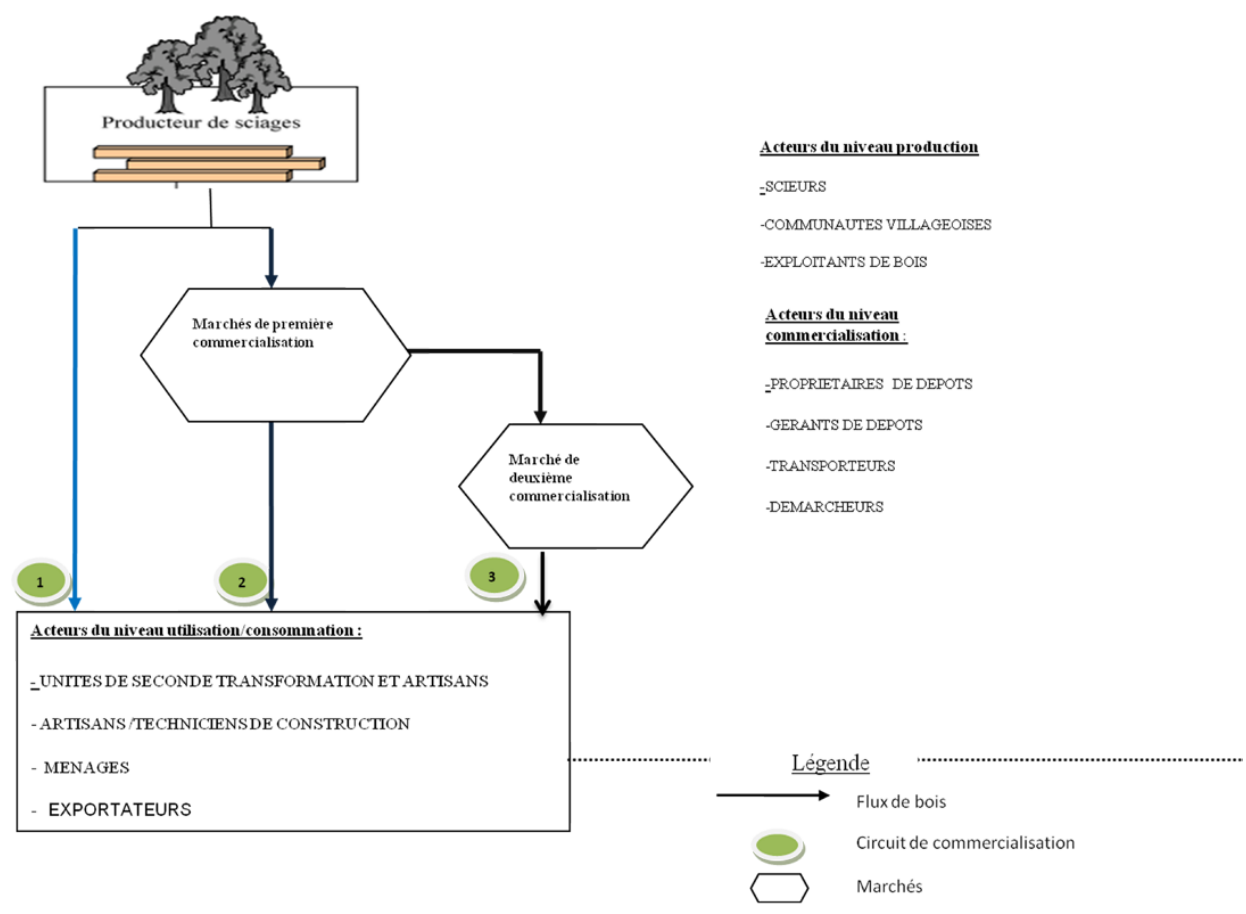

Figure 1: Les trois circuits et principaux acteurs de la commercialisation du bois de sciage artisanal dans le marché domestique camerounais

Identification des facteurs de compétitivité à partir de l'analyse des stratégies de produits et prix dans le marché intérieur du bois de sciage artisanal

Les tableaux $4,5,6$, et 7 ci-dessous présentent une photographie de la structuration ou de la stratégie de l'offre en termes de produit et de prix dans 4 marchés urbains étudiés. On doit déjà noter qu'il n'y a aucune interconnexion entre les marchés des villes de Kribi, Sangmélima et Ebolowa. Les prix se fixent suivant le niveau de l'offre et de la demande et sur base des qualités physico-techniques du bois ; il est donc logique et facile à comprendre que la ville de Kribi qui est plus proche de Douala et connaît une demande plus forte que celle de Sangmélima offre des prix différents de ceux de Sangmélima pour certain bois précieux comme le Bubinga. 


\begin{tabular}{|c|c|c|c|c|}
\hline Catégorie & Essences (nom scientifique) & $\begin{array}{l}\text { Désignation } \\
\text { pièce }\end{array}$ & Prix/Pièce & Prix $/ \mathrm{m}^{3}$ \\
\hline \multirow[t]{2}{*}{ Haut de gamme } & Bubinga (Guibourtia tessmannii) & Planche & 6.500 & 128.000 \\
\hline & $\begin{array}{l}\text { Moabi (Baillonnella toxisperma), } \\
\text { Doussié (Afzelia bipindensis), } \\
\text { Pachy (Afzelia pachyloba) }\end{array}$ & Planche & 4.000 & II 4.000 \\
\hline Moyen de gamme & $\begin{array}{l}\text { Bossé clair (Guarea cedrata), } \\
\text { Bilinga (Nauclea diderrichii), } \\
\text { Iroko (Milicia excelsa), } \\
\text { Padouk (Pterocarpus soyauxii), } \\
\text { Movingui (Distemonanthus } \\
\text { benthamianus), } \\
\text { Bibolo (Lovoa trichilioides) }\end{array}$ & Planche & 3.000 & 85.000 \\
\hline \multirow[t]{6}{*}{ Bas de gamme } & \multirow{3}{*}{$\begin{array}{l}\text { Ayous (Triplochiton scleroxylon), } \\
\text { Frakè (Terminalia superba) }\end{array}$} & Lattes & I.000 & 62.500 \\
\hline & & Planche & 2.500 & 55.500 \\
\hline & & Chevron & I.500 & 47.000 \\
\hline & Bongo (Fagara buesgenii) & Basting & 4.000 & 52.000 \\
\hline & Ekop (Librevillia klainei) & Basting & $4 \cdot 500$ & 59.000 \\
\hline & $\begin{array}{l}\text { Dabema (Piptadeniastrum } \\
\text { africanum) }\end{array}$ & Basting & $3 \cdot 500$ & 46.000 \\
\hline
\end{tabular}

Tableau 4: Offre produits-prix (en FCFA) dans le marché de Kribi (Source: données de notre étude) 


\begin{tabular}{|c|c|c|c|c|}
\hline Catégorie & $\begin{array}{l}\text { Essences } \\
\text { (nom commercial local) }\end{array}$ & Désignation & Prix/Pièce & Prix $/ \mathrm{m}^{3}$ \\
\hline \multirow[t]{2}{*}{ Haut de gamme } & Bubinga (Guibourtia tessmannii) & Planche & $4 \cdot 500$ & I28.000 \\
\hline & Moabi (Baillonnella toxisperma) & Planche & 3.000 & 85.000 \\
\hline Moyen de gamme & $\begin{array}{l}\text { Sapelli (Entandrophragma } \\
\text { cylindricum), } \\
\text { Bilinga (Nauclea diderrichii), } \\
\text { Iroko (Milicia excelsa), } \\
\text { Padouk (Pterocarpus soyauxii), } \\
\text { Movingui (Distemonanthus } \\
\text { benthamianus), } \\
\text { Bibolo (Lovoa trichilioides), } \\
\text { Kossipo (Entandrophragma } \\
\text { candollei) }\end{array}$ & Planche & 2.500 & 71.000 \\
\hline \multirow[t]{3}{*}{ Bas de gamme } & \multirow{3}{*}{$\begin{array}{l}\text { Ayous (Triplochiton scleroxylon) } \\
\text { Frakè (Terminalia superba), } \\
\text { Eyong (Eribroma oblongum) }\end{array}$} & Planche & 2.200 & 49.000 \\
\hline & & Chevron & I. 500 & 47.000 \\
\hline & & Lattes & 800 & 50.000 \\
\hline
\end{tabular}

Tableau 5: Offre produits-prix (en FCFA) dans le marché de Sangmélima (Source: données de notre étude) 


\begin{tabular}{|c|c|c|c|c|}
\hline Catégorie & $\begin{array}{l}\text { Essences } \\
\text { (nom commercial local et nom } \\
\text { scientifique) }\end{array}$ & $\begin{array}{l}\text { Désignation/ } \\
\text { Pièce }\end{array}$ & Prix/Pièce & $\mathrm{P} . \mathrm{V} / \mathrm{m}^{3}$ \\
\hline \multirow[t]{3}{*}{ Haut de gamme } & Wengué (Millettia laurenti) & Planche & 10.000 & 84.000 \\
\hline & Bubinga (Guibourtia tessmannii) & Planche & I0.000 & 84.000 \\
\hline & $\begin{array}{l}\text { Moabi (Baillonnella toxisperma), } \\
\text { Doussié (Afzelia bipindensis) }\end{array}$ & Planche & 5.000 & $\mathrm{I} 42.000$ \\
\hline Moyen de gamme & $\begin{array}{l}\text { Pachy (Afzelia Pachyloba), Bete } \\
\text { (Mansonia altissima), Acajou (Khaya } \\
\text { ivorensis), Makoré (Tieghemella } \\
\text { africana), Zingana (Microberlinia } \\
\text { brazzavillensis), Bossé clair (Guarea } \\
\text { cedrata), Bilinga (Nauclea diderrichii), } \\
\text { Iroko (Milicia excelsa), Padouk } \\
\text { (Pterocarpus soyauxii), Movingui } \\
\text { (Distemonanthus benthamianus), } \\
\text { Bibolo (Lovoa trichilioides), } \\
\text { Sapelli (Entandrophragma } \\
\text { cylindricum), Kossipo } \\
\text { (Entandrophragma candollei) }\end{array}$ & Planche & 4.000 & II4.000 \\
\hline \multirow[t]{6}{*}{ Bas de gamme } & Ayous (Triplochiton scleroxylon) & Planche & 2.500 & $55 \cdot 500$ \\
\hline & Frakè (Terminalia superba) & Chevron & I. 500 & 47.000 \\
\hline & \multirow{4}{*}{$\begin{array}{l}\text { Eyong (Eribroma oblongum) } \\
\text { Dabema (Piptadeniastrum } \\
\text { africanum) } \\
\text { Bahia (Mitragyna ciliata) }\end{array}$} & Lattes & I.000 & 62.500 \\
\hline & & Basting & 4.000 & 52.000 \\
\hline & & Basting & 4.500 & 59.000 \\
\hline & & Basting & $4 \cdot 500$ & 59.000 \\
\hline
\end{tabular}

Tableau 6: Les produits et prix (en FCFA) dans le marché de Yaoundé (Source: données de notre étude) 


\begin{tabular}{|c|c|c|c|c|}
\hline Catégorie & $\begin{array}{l}\text { Essences } \\
\text { (nom commercial local) }\end{array}$ & Désignation & Prix/Pièce & Prix $/ \mathrm{m}^{3}$ \\
\hline \multirow[t]{7}{*}{ Haut de gamme } & Bubinga (Guibourtia tessmannii) & Planche & I2.500 & 355.000 \\
\hline & Doussié (Afzelia bipindensis) & Planche & 9.000 & 256.000 \\
\hline & $\begin{array}{l}\text { Mukulungu (Autranella } \\
\text { congolensis) }\end{array}$ & Planche & 6.500 & 246.000 \\
\hline & $\begin{array}{l}\text { Zingana (Microberlinia } \\
\text { brazzavillensis) }\end{array}$ & Planche & 8.000 & 227.500 \\
\hline & Pachy (Afzelia Pachyloba) & Planche & $7 \cdot 500$ & 213.000 \\
\hline & Moabi (Baillonnella toxisperma) & Planche & 6.500 & 185.000 \\
\hline & Bossé Clair (Guarea cedrata) & Planche & 6.000 & 170.000 \\
\hline \multirow[t]{3}{*}{ Moyen de gamme } & $\begin{array}{l}\text { Bongo (Fagara buesgenii), } \\
\text { Bilinga (Nauclea diderrichii), } \\
\text { Iroko (Milicia excelsasa) } \\
\text { Padouk (Pterocarpus soyauxii) } \\
\text { Movingui (Distemonanthus } \\
\text { benthamianus) } \\
\text { Bibolo (Lovoa trichilioides) } \\
\text { Sapelli (Entandrophragma } \\
\text { cylindricum) } \\
\text { Kossipo ( Entandrophragma } \\
\text { candollei) }\end{array}$ & Planche & 5.000 & 142.000 \\
\hline & Azobé (Lophira alata) & Planche & 5.280 & 150.000 \\
\hline & Tali (Erythropleum ivorense) & Planche & 5.280 & 150.000 \\
\hline \multirow[t]{3}{*}{ Bas de gamme } & $\begin{array}{l}\text { Ayous (Triplochiton scleroxylon) } \\
\text { Frakè (Terminalia superba) } \\
\text { Eyong (Eribroma oblongum) }\end{array}$ & Planche & 3.800 & 84.500 \\
\hline & $\begin{array}{l}\text { Dabema (Piptadeniastrum } \\
\text { africanum) }\end{array}$ & Chevron & 2.600 & 82.000 \\
\hline & Frakè (Terminalia superba) & Lattes & I.000 & 62.500 \\
\hline
\end{tabular}

Tableau 7: Les produits et prix (en FCFA) dans le marché de Douala (Source: données de notre étude)

De l'analyse qualitative de ces données, il ressort que l'offre dans les grandes villes (Yaoundé et Douala) présente à la fois des différences et des points de similitudes avec celle des petites villes.

Comme différences, on observe que :

- l'offre dans les grandes villes est plus diversifiée en termes d'essences (une vingtaine contre une dizaine dans les petites villes). Ceci traduit une demande plus dynamique dans les grands centres urbains comme nous l'avons déjà évoqué plus haut; et

- les prix pratiqués dans les grandes villes, pour ce qui est des planches, sont parfois 
deux fois plus élevés dans les grands centres urbains de Douala et Yaoundé. Ceci s'explique par l'éloignement des points d'approvisionnement dans ces 2 villes. En plus, certaines essences arrivant à Douala transitent d'abord par Yaoundé. Ce qui rend les prix encore plus élevés dans cette première ville.

Comme similitudes, on observe que:

- tous les marchés présentent 3 niveaux dans leur gamme de produit, traduisant une différenciation en termes de qualité dans les marchés. Les planches issues des essences telles que le Bubinga (Guirboutia tessmanii) et le Moabi (Baillonela toxisperma) seraient plus prisées sur le marché en raison de leurs caractéristiques physiques, les croyances magico religieuses et les vertus diverses qui leurs sont associées, les coûts relativement élevés d'exploitation (sciage laborieux, transport difficile) d'où leurs prix relativement élevés par rapport aux planches d'autres essences ;

- les essences proposées en bas de gamme ont des prix sensiblement identiques dans tous les marchés. En raison d'une forte concurrence des déchets de scieries industrielles à ce niveau de la gamme de produits, les vendeurs des grands centres urbains ont développé une stratégie d'approvisionnement de proximité afin d'offrir sur le marché des produits à des prix compétitifs pour le consommateur. Ce dernier aspect de similitude fait ressortir la logique de compétition sur les coûts de revient en matière de production et de vente des produits.

Sur la base de ces deux derniers points de similitudes, nous relevons qu'un producteur peut développer sa compétitivité sur le marché du bois de sciage artisanal en mettant en œuvre l'une ou l'ensemble des deux stratégies suivantes:

- stratégie de qualité, basée sur des essences prisées et rares, lui permettant ainsi de pratiquer des prix plus élevés et très rentables; et/ou

- stratégie de coût en offrant sur le marché des essences abondantes à un coût avantageux, lui permettant ainsi de résister à la concurrence sur les prix.

Dans les paragraphes qui suivent, nous allons, à partir de l'analyse de la chaîne de valeur de production, présenter les points faibles des forêts communautaires par rapport à la concurrence. 
La compétitivité des forêts communautaires au niveau de la qualité des essences et des coûts de mise en marché du bois de sciage artisanal

Comme présentées dans la figure 2 ci-dessous, cinq principales opérations sont nécessaires pour la vente du bois de sciage artisanal.
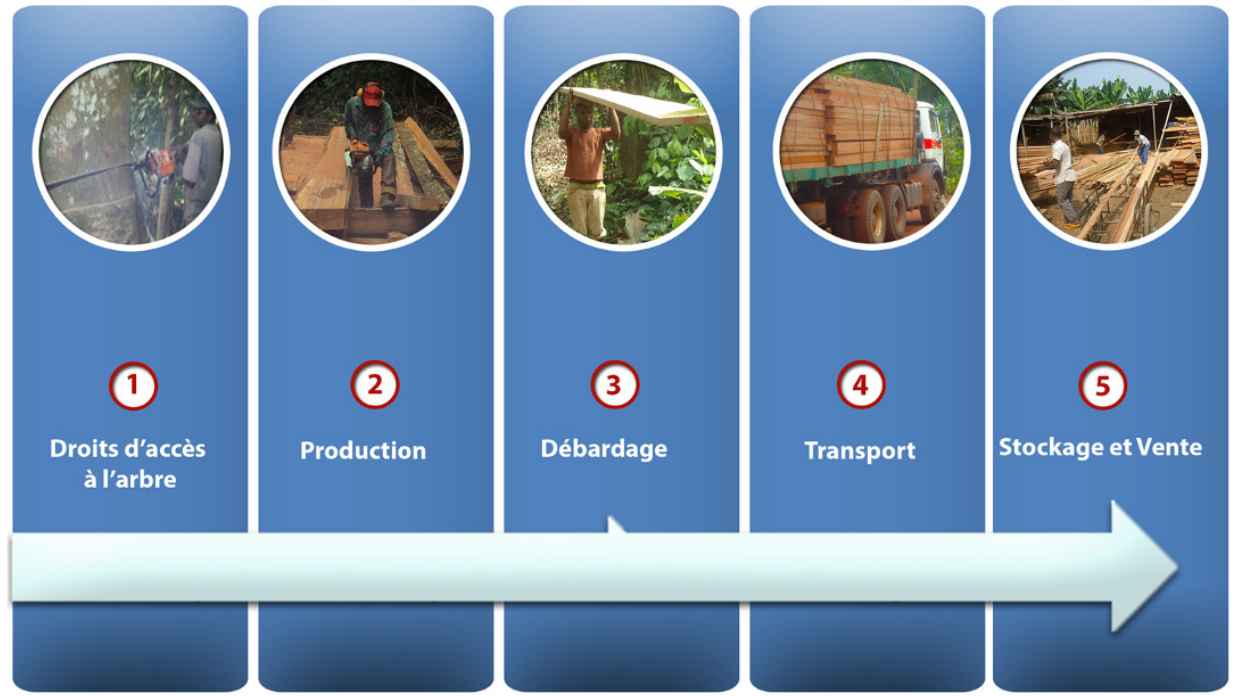

Figure 2 : Le processus de création de la valeur dans le marché local du bois d'œuvre

(I) Droit d'accès à l'arbre: pour les acteurs du sciage informel, il s'agit de payer une certaine somme d'argent au "propriétaire" d'un arbre sur pied afin de le couper. Ceci est cependant une coupe illicite, dans la mesure où la Loi forestière de Janvier I994 au Cameroun stipule que les arbres appartiennent à l'Etat. Il reconnaît dans son article 8 le droit d'usage aux riverains des espaces forestiers et non un droit de vente des arbres. Par contre, pour les gestionnaires de FC, elles doivent justifier d'un inventaire réalisé sur une parcelle de leur FC, laquelle est validée par le gouvernement qui délivre par la suite un certificat annuel d'exploitation (CAE), précisant les essences et les volumes autorisées à l'exploitation.

(2) Production: s'agissant de la production, c'est un ensemble d'activités qui permettent d'abattre et de débiter l'arbre sous forme de pièces de dimensions requises pour le marché. Elles impliquent l'utilisation d'un ensemble d'équipements (tronçonneuses, Scies mobiles), des intrants de production (carburants, lubrifiants, consommables mécaniques), de la main d'œuvre, ainsi qu'une capacité de planification technique et financière de la production.

(3) Débardage: le débardage est l'ensemble des activités permettant le transport de pièces débitées du lieu de production à l'endroit où il sera accessible aux véhicules appropriés de transport. Il requiert une main d'œuvre importante capable d'effectuer le transport, généralement par tête pour les différents opérateurs, sachant que 
les engins de débardage de grandes dimensions sont proscrits dans les forêts communautaires (MINFOF, 2009).

(4) Transport: le transport est l'opération d'évacuation des pièces de bois des localités de production vers les marchés locaux ou nationaux où elles pourront être vendues.

(5) Stockage : le stockage et vente renvoient à l'ensemble des activités de stockage, de promotion et de distribution du produit dans les marchés. Il requiert l'accès aux points de ventes appropriés dans les villes de consommation.

Si l'on s'en tient à la chaîne de valeur du bois de sciage artisanal, présentée ci-dessus, la compétitivité sur la qualité au niveau des FC ne peut se faire qu'à travers les parcelles qui leur sont attribuées par l'Etat. Ces parcelles sont-elles suffisamment fournies en essences de qualité leur permettant d'être compétitives sur le marché? Les tableaux 8 et 9 ci-dessous nous présentent les données d'analyse de l'offre potentielle en termes de qualité de II FC.

\begin{tabular}{lr|r|r|r|r}
\hline Produits & GESFOCTECH & SOLIDARITE & GICAN II & ABAPI & APMI \\
\hline $\begin{array}{l}\text { Proportion des essences } \\
\text { de hautes et moyennes } \\
\text { qualités (en \%) }\end{array}$ & I4 & I5 & 28 & I4 & I5 \\
\hline $\begin{array}{l}\text { Volume totale de bois } \\
\text { disponible }\left(\mathrm{en} \mathrm{m}^{3} \text { ) }\right.\end{array}$ & 28.207 & 30.374 & $32.58 \mathrm{I}$ & 194.944 & III.746 \\
\hline
\end{tabular}

Tableau 8: Profil d'offre potentielle en qualité des forêts communautaires du Dja et Lobo (Source: données de notre étude)

\begin{tabular}{lr|r|r|r|r|r}
\hline Produits & RDFCAB & UCFA & OTONON & APM & ALMA & ACOMINE \\
\hline $\begin{array}{l}\text { Proportion des essences } \\
\text { de hautes et moyennes } \\
\text { qualités (en \%) }\end{array}$ & 24 & I5 & I3 & 5 & 27 & 48 \\
\hline $\begin{array}{l}\text { Volume totale de bois } \\
\text { disponible }\left(\mathrm{en} \mathrm{m}^{3} \text { ) }\right.\end{array}$ & 47.034 & 229.939 & I88.404 & 58.500 & 52.233 & 18.257 \\
\hline
\end{tabular}

Tableau 9: Profil d'offre potentielle en qualité des forêts communautaires de l'Océan et de la Vallée du Ntem

De l'analyse des tableaux 8 et 9 , il ressort ce qui suit:

- $\quad$ il y'a une disparité importante entre les FC en termes de volume total de bois et de proportion des essences de qualité. S'agissant du premier aspect de comparaison, l'intervalle se situe entre $28.207 \mathrm{~m} 3$ pour le volume le plus bas et I94.944 m3. Pour ce qui est de la proportion des essences de qualité, l'intervalle se situe entre $5 \%$ pour la proportion la plus basse et $48 \%$ pour la proportion la plus haute;

- dans tous les cas étudiés, les forêts communautaires sont peu fournies en essences de haute ou moyenne qualité. Ces essences représentent moins de la moitié des volumes attribuées aux FC, les privant ainsi d'un avantage réel en termes de qualité 
des essences. Cette faible densité s'explique par le fait que, conformément à la Loi forestière camerounaise, les forêts communautaires sont attribuées dans le DFnP, lequel a subi, très souvent par le passé, une exploitation forestière intense et très sélective de la part des industriels.

Sur le plan du contrôle de l'espace de coupe, la totalité des FC au stade d'exploitation, dont disposant d'une convention définitive de gestion, ne représente que 790.794,9 ha (MINFOF, 20II), soit une infirme proportion des DFnP. Le nombre d'initiatives de forêts communautaires déjà attribuées ne représentaient que 21\% du DFnP en 2010, et s'il arrivait que toutes les I.45I demandes et initiatives en cours de FC atteignent la phase d'exploitation, elles ne représenteront que 34\% du DFnP (Cuny, 20II). Autrement dit, les opérateurs du secteur informel ont encore une bonne marge de manœuvre d'approvisionnement en essences de qualité sur les espaces non aménagés du DFnP.

En se basant sur les opérations de la chaîne de valeur présentées plus haut, la figure 3 met en évidence la structure de coût de mise en marché d'une essence de qualité par les opérateurs du secteur informel et les FC. Il ressort de l'analyse de celle-ci les éléments qui suivent:

- le coût moyen de mise en marché d'un arbre dans une forêt communautaire se situe en moyenne autour de FCFA 7I.3Io (soit environ €I08,7I) par $\mathrm{m}^{3}$, tandis qu'il est de FCFA 57.300 (soit environ $€ 87$ ) par $\mathrm{m}^{3}$ pour un opérateur illégal, soit une différence de près de $25 \%$;

- le coût de la main d'œuvre de production est moins élevé dans les FC que chez les opérateurs informels. Ces derniers déplacent généralement des travailleurs du centre urbain pour les villages, ce qui alourdit les charges de la main d'œuvre;

\section{Coût (en FCFA/m3)}

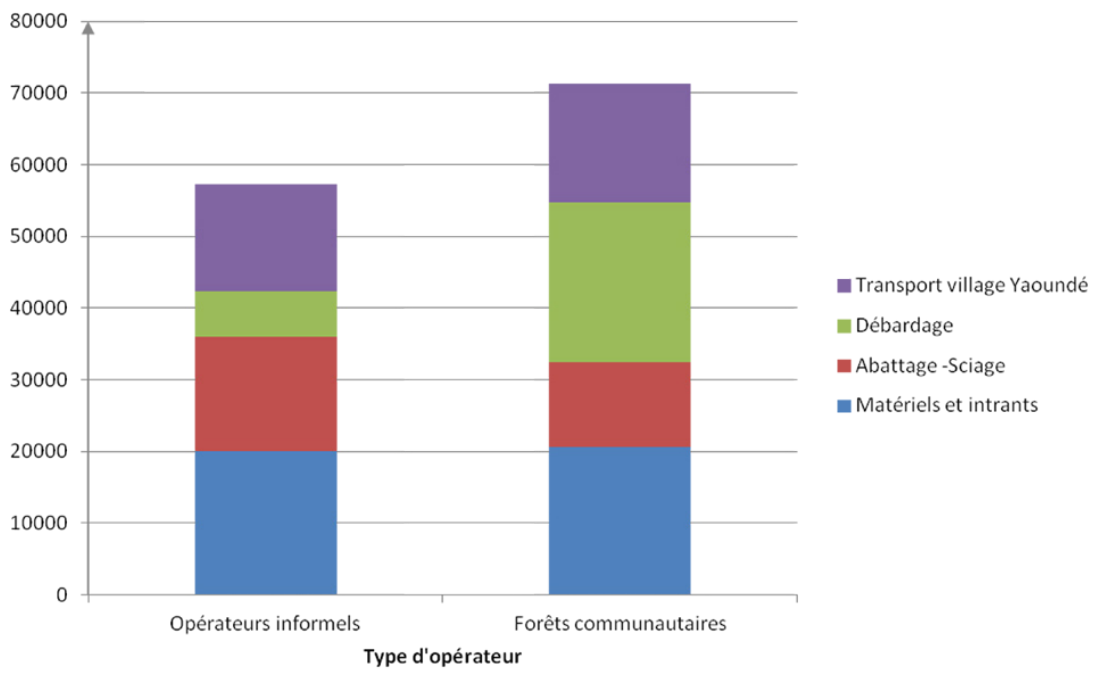

Figure 3: Analyse comparative de la structure de coût de mise en marché du bois par les opérateurs du secteur informels et les forêts communautaires dans la Région du Sud Cameroun 
- le coût de débardage est près de 2 fois plus élevés chez les FC que chez les opérateurs informels ; il est estimé en moyenne à FCFA 22.350 (soit $€_{34,072)}$ par m³ $^{3}$ pour les premiers contre FCFA 6.300 (soit $€_{9,6}, 6$ ) par $m_{3}$ pour les seconds. En effet, les FC doivent respecter un plan d'aménagement de l'espace qui leur est attribué, les obligeant à couper dans des parcelles très éloignées des points d'évacuation pour la ville. Chez les opérateurs illégaux, la proximité d'un arbre par rapport au point d'évacuation est déterminante pour son exploitation. Ces deux logiques différentes d'exploitation expliquent en grande partie ce différentiel de coût de mise en marché entre les FC et les opérateurs illégaux;

- le coût de transport du village vers Yaoundé est sensiblement le même pour les deux types d'opérateurs. Cela s'explique par le fait que tous les deux utilisent le même moyen d'évacuation de produits vers les grands centres urbains.

En définitive, la maitrise des coûts de production et surtout du débardage du bois est une source d'avantage importante pour les producteurs de bois sciés. Et à ce jour, les FC sont considérablement désavantagées au niveau de cette dernière opération de la chaîne de valeur du bois de sciage artisanal.

\section{Conclusions et perspectives pour la compétitivité des forêts communautaires}

L'étude sur la compétitivité des FC sur le marché de bois de sciage artisanal a permis de mettre en évidence les facteurs de compétitivité et la position des FC sur le marché de sciage artisanal au Cameroun. Il en ressort des interviews avec I2 FC dans la Région du Sud et des études qualitatives de marché dans 5 villes du Cameroun, que la qualité des essences et la maitrise des coûts de production sont deux facteurs déterminants de la compétitivité de l'offre sur le marché de bois de sciage artisanal. Par rapport à ces deux facteurs de compétitivité, les FC sont actuellement très mal positionnées pour faire une concurrence gagnante avec les opérateurs du secteur informel. D'une part les surfaces qu'elles exploitent dans le DFnP sont très peu denses en essences de qualité, et d'autre part, du fait des contraintes de débardage plus complexe, leur coût de mise en marché du bois est près de $25 \%$ plus élevé que celui des opérateurs du secteur informel. On peut dès lors comprendre le faible niveau d'exploitation et la tendance à la fraude dans certaines FC. Si l'Etat et les partenaires au développement souhaitent véritablement dynamiser l'exploitation du bois dans les FC et leur permettre de contribuer effectivement à la lutte contre la pauvreté en milieu rural, plusieurs actions doivent être entreprises.

A défaut de prendre des mesures rigoureuses pour freiner l'exploitation illégale (ce qui est très difficile au regard du contexte de gouvernance dans les pays de l'Afrique centrale comme le Cameroun et de la nécessité de satisfaire des besoins importants et croissants des grands centres urbains à très court terme) ou alors d'encourager les communautés vers le marché export (qui est difficilement accessible à l'heure actuelle au regard des contraintes technico-financières, et la faible disponibilité des essences exports dans les FC), les actions suivantes sont recommandées : 
- à court terme, il faut: (I) accompagner les communautés dans l'exploitation d'autres produits de leurs agro-forêts (fruits, miels, écorces, lianes, bambou ...) ; pour cela le gouvernement à travers le MINFOF doit exiger, au moment de l'attribution des FC, que des études techniques et financières sur l'exploitation de ressources variées de la FC soient effectuées afin de déterminer celles qui seront facilement exploitables à court terme par la communauté.

- à moyen et long terme, il faut: (2) soutenir les communautés dans l'enrichissement des parcelles en essences de qualité à travers la création et la gestion des pépinières communautaires ; (3) encourager la recherche sur les techniques moins coûteuses de production et débardage du bois dans les chantiers d'exploitations artisanales; (4) faciliter la promotion des essences peu utilisées dans le secteur de la seconde transformation en établissant un lien entre les menuiseries et les communautés, avec le soutien des structures de recherche; (5) accompagner quelques initiatives pilotes de commerce du carbone dans le cadre du mécanisme REDD+, surtout dans le cas des FC très enclavées. Les recommandations (3), (4) et (5) sont actuellement mises en œuvre par le WWF dans le cadre des projets DACEFI 2 et REDD+ NGOYLA MINTOM, tous financés par la Commission Européenne.

\section{Références}

Adja, Mirango \& Nana, Tayou (2007). Etude de caractérisation des forêts communautaires de la province de l'Est, SNV/MINFOF, Yaoundé, Cameroun.

Bakouma, J. \& Sève, J. (2012). La gestion forestière par l'entreprise communautaire. Secteur privéet Développement (14), Proparco, Mai 2012, P.13.

Cerrutti, P.O. \& Lescuyer, G. (20II). Le marché domestique du sciage artisanal au Cameroun, états des lieux, opportunités et défis, Document Occasionnel (59), CIFOR, Bogor, Indonesie, 56 p.

Cuny, P. (20II). Etats de lieux de la foresterie communautaire et communale au Cameroun, Tropenbos International Programme du bassin du Congo, Wageningen, Pays bas, xviii+ıro p.

ECAM 2 (2003). Deuxième Enquête Camerounaise Auprès des Ménages, MINEFI/Direction de la statistique et de la comptabilité nationale, Yaoundé, Cameroun.

ECAM 3 (2008). Troisième Enquête Camerounaise Auprès des ménages, MINEFI/Direction de la statistique et de la comptabilité nationale, Yaoundé, Cameroun.

Ingram, V., Beauchamp, E., Lescuyer, G., Parren, M., Njomgang, C., Awono, A. (2010). Costs, benefits and impacts of community forests on livelihoods in Cameroon, in Taking stock of smallholder and community forestry: Where do we go from here?, Atelier international à Montpellier, France, 24 - 26 mars 2010.

Julve, C., Vandenhaute, M., Vermeulen, C., Castadot, B., Ekodeck, H., Delvingt, W. (2007). Séduisante théorie, douloureuse pratique, la foresterie communautaire en butte à sa propre législation, In Parcs et Réserves, Volume 62 (2), p. I8.

Kana, R., Ondoua, B., Etoga, G. (20II). La promotion des initiatives de foresterie communautaire autour du parc national de Campo Ma'an, Approches, Résultats, Défis, Programme WWF Kudu Zombo, Cameroun.

Koffi Yeboa, A.N. (2005). Sciage artisanal, transformation et commerce du bois d'œuvre du Cameroun à destination de l'arc soudano-sahélien. Mémoire de fin d'étude, ENGREF, Nancy, France.

Kotler, P., Dubois, B., Keller, K, Manceau, D. (2006). Marketing Management. Paris : I2e édition, éd. Pearson Education, 5 II p. 
Lambin, J.J. et Chumpitaz, R. (2002). Marketing stratégique et opérationnel, du marketing à l'orientation marché. Paris : 5 e eds, Dunod, 517 p.

Lendrevie, J. et Lindon, D. (2000). Mercator, Théorie et Pratique du marketing. Paris : 6e edition, Dalloz, eds, $755 \mathrm{p}$.

Lescuyer, G. (2005). Formes d'action collective pour la gestion locale de la forêt camerounaise, organisations « modernes " ou institutions « traditionnelles ", in Vertigo, I6 (3), p.84.

Lescuyer, G., Eba'a Atyi, R., Cerutti, P. (2009). Consommations nationales de bois d'œuvre en Afrique Centrale, enjeux majeur pour la gestion forestière durable, XIII Congrès forestier mondial, Buenos Aires, Argentina, I8-23 Octobre 2009.

MINFOF (2009). Manuel des procédures d'attribution et normes de gestion des forêts communautaires au Cameroun, Yaoundé, Cameroun.

MINFOF (20I2). Rapport de l'atelier bilan sur la foresterie communautaire au Cameroun, du I3-I4 Décembre 20 II à Ebolowa, Cameroun.

Nzoyem Maffo, H.N., Vabi, M., Kouokam, R., Azanga, C. (2010). Forêts communautaires contre la pauvreté, la déforestation et la dégradation des forêts : en faire une réalité au Cameroun, in "Taking stock of smallholder and community forestry: Where do we go from here?", atelier international à Montpellier, France, 24 - 26 mars 2010.

Plouvier, D., Eba'a Atyi, R., Fouda, T., Oyono, R., Djeukam, R. (2002). Etude du sous-secteur sciage artisanal au Cameroun. Yaoundé, Cameroon, Ministry of Environment and Forests.

République du Cameroun (I994). Loi N94/01 du 20 Janvier 1994 portant régime des forêts, de la faune et de la pêche.

Sonne, N., Ekodeck, H., Ondoua, B., Tayo, C., (20II). Foresterie communautaire au Cameroun, expériences et contributions du WWF (2003-2010), Yaoundé, WWF-Programme Cameroun.

Stanton, W., (I964). Fundamentals of marketing, New York: Ist edition, McGrawhill Book Company, 6oo p. 